\title{
ASSISTÊNCIA INCLUSIVA E CONSTRUÇÃO DE EDUCAÇÃO MÉDICA: UM RELATO DE EXPERIÊNCIA DE UM FÓRUM ONLINE SOBRE A SAÚDE LGBTQIA+
}

\begin{abstract}
Fernanda Clara da Silva1, ORCID ID 0000-0002-8030-7621; Sadi Bruno Freitas Santin', ORCID ID 0000-00034859-0057; Ellany Gurgel Cosme do Nascimento2; ORCID ID 0000-0003-4014-6242
\end{abstract}

\section{FILIAÇÃO}

(1) Universidade do Estado do Rio Grande do Norte, Faculdade de Ciências da Saúde (FACS/UERN), Departamento de Ciências Biomédicas, Discente de Medicina

(2) Universidade do Estado do Rio Grande do Norte, Faculdade de Ciências da Saúde (FACS/UERN), Departamento de Ciências Biomédicas, Doutora em Ciências da Saúde, Docente da disciplina de Epidemiologia

\section{AUTOR CORRESPONDENTE}

Fernanda Clara da Silva; fernandaclara@alu.uern.br; Universidade do Estado do Rio Grande do Norte, Faculdade de Ciências da Saúde (FACS/UERN); discente de Medicina; Endereço: Rua Antonio Vieira de Sá, 1840. Ap 07. Bairro Aeroporto I. Mossoró/RN. CEP 59607-100.

\section{MENSAGENS-CHAVE}

\section{Contextualização sobre o tema}

A saúde da população LGBTQIA+ é historicamente marginalizada e, no campo da saúde, isso é ainda mais grave. Assim, é importante discutir a visibilidade médica da pessoa LGBTQIA+.

\section{Novas descobertas}

A maior parte dos participantes do Proud by Nature - I Fórum de Saúde LGBTQIA+ afirmou não ter contato com a temática antes do evento e se avaliaram com conhecimentos insuficientes para atender as demandas LGBTQIA+.

\section{Implicações das novas descobertas}

É preciso suscitar o debate na comunidade acadêmica sobre a pauta LGBTQIA+, já que se nota carência desse conteúdo desde a graduação dos cursos da saúde e que se perpetua durante a prática nos serviços médicos.

\section{Consequências dos achados}

É relevante tratar da atual negligência à saúde do grupo LGBTQIA+ realizando reformas curriculares, programas, eventos que tratem cada vez mais do tema, como foi este evento.

\section{RESUMO}

INTRODUÇÃO: A saúde da comunidade LGBTQIA+, enquanto bem-estar físico e mental, só é efetiva quando garante o acesso integral na saúde desses indivíduos. Embora o Sistema Único de Saúde (SUS) tenha implementado avanços na visibilidade desse grupo marginalizado, existem diversas especificidades e demandas referentes à essa população que são perpetuadas não só pelo preconceito, mas também pela deficiência advinda de currículos não plurais nas graduações dos cursos de saúde. Assim, o objetivo do trabalho é relatar a experiência de um evento voltado para a saúde LGBTQIA+, e demonstrar a importância e os impactos da discussão dessa pauta na comunidade acadêmica. RELATO: O "Proud by Nature I Fórum de Saúde LGBTQIA+", foi um evento realizado totalmente de forma online, pensado principalmente para profissionais e estudantes das diversas área da saúde a fim de suscitar o debate sobre a saúde dessa comunidade, uma pauta tão importante e negligenciada, abordando temas como: saúde sexual, prevenção de ISTs, atendimento médico humanizado, 
linguagem inclusiva e saúde mental. DISCUSSÃO: Diante do exposto, percebe-se que há resistência do público LGBTQIA+ em procurar serviços de saúde, principalmente da população trans em ter atendimento específico. Além disso, a exposição à violência e ao preconceito deixam a saúde mental desse grupo vulnerável. Ainda, há baixa adesão às políticas públicas de sexo seguro e prevenção de ISTs, o que impacta na falta de informação básica em prevenção, diagnóstico e tratamento. Por fim, é importante destacar que a luta LGBTQIA+ pelos direitos básicos, como a saúde, precisa continuar e ser garantida. CONCLUSÃO: Os objetivos propostos pelo evento foram cumpridos. Ainda, demonstra-se que as instituições de ensino superior de medicina e suas entidades têm um papel transformador na contribuição não só com a comunidade acadêmica, mas também com o público exterior, espalhando conhecimento em conteúdos muitas vezes velados, como é a saúde LGBTQIA+.

PALAVRAS-CHAVE: Educação em saúde; Humanização da assistência; Minorias sexuais e de gênero.

\begin{abstract}
INTRODUCTION: The health of the LGBTQIA+ community, as physical and mental well-being, is only effective when it guarantees full access to the health of these individuals. Although the Unified Health System (SUS) has implemented advances in the visibility of this marginalized group, there are several specificities and demands regarding this population that are perpetuated not only by prejudice, but also by the deficiency arising from non-plural curricula in the graduation of health courses. Thus, the objective of the work is to report the experience of an event focused on LGBTQIA+ health, and demonstrate the importance and impacts of discussing this agenda in the academic community. REPORT: The "Proud by Nature - I Forum on LGBTQIA+ Health", was an event held entirely online, being designed mainly for professionals and students in the various areas of health in order to raise the debate on such an important and neglected agenda that it is the health of this community, covering topics such as: sexual health, prevention of STIs, humanized medical care, inclusive language and mental health. DISCUSSION: Is clear that there is resistance from the LGBTQIA+ public to seek health services, especially from the trans population to have specific care. In addition, exposure to violence and prejudice makes the mental health of this group vulnerable. Furthermore, there is low adherence to public policies on safe sex and prevention of STIs, which impacts the lack of basic information on prevention, diagnosis and treatment. Finally, it is important to highlight that the LGBTQIA+ fight for basic rights, such as health, needs to continue and be guaranteed. CONCLUSION: The objectives proposed by the event were fulfilled. Still, it is shown that higher education institutions of medicine and their entities have a transforming role in contributing not only to the academic community, but also to the outside public, spreading knowledge in often veiled contents, such as LGBTQIA+ health.
\end{abstract}

\title{
KEYWORDS: Health education; Humanization of assistance; Sexual and gender minorities.
}

\section{INTRODUÇÃO}

De acordo com a Constituição Federal Brasileira (1988), a saúde é um direito de todos e dever do Estado, com validade sem qualquer tipo de discriminação (1). Entretanto, longe dos papéis, sabe-se que essa determinação não ocorre de forma tão igualitária, espelhando quadros de desigualdade e exclusão de grupos minoritários, seja por raça, gênero, sexualidade, dentre outros. Em consonância com essa determinante, compreende-se uma sociedade conservadora marcada pelo patriarcado e pela cultura predominante heterocisnormativa, que culmina no tratamento desigual, inclusive nos serviços de saúde. Nesses espaços, Lésbicas, Gays, Bissexuais, Transexuais, Travestis, Queer, Intersexuais, Assexuais e outros (LGBTQIA+) são um dos grupos mais atingidos por essa dessemelhança social e cultural (2).

Nessa perspectiva, a saúde da comunidade LGBTQIA+, enquanto bem-estar físico e mental, só é efetiva quando há quebra das barreiras das desigualdades coletivas, a fim de garantir a atenção e acesso integral à saúde de um indivíduo. Para tal, mobilizações nos âmbitos políticos, econômicos, culturais, psicológicos e comportamentais - ou seja, Determinantes Sociais da Saúde (DSS) - se fazem indispensáveis no intuito de coibir as iniquidades (3). No
Brasil, a Política Nacional de Saúde Integral de Lésbicas, Gays, Bissexuais, Travestis e Transexuais (PNSILGBT, 2011) e o Processo Transexualizador (2008) foram implementados no Sistema Único de Saúde (SUS), representando um avanço nesta pauta e dando visibilidade a esse grupo marginalizado. Embora mais medidas sejam essenciais na garantia do direito à saúde, tais políticas corroboram com os princípios de universalidade, integralidade e equidade (4).

Com base nessa conjuntura, nota-se que existem diversas especificidades e demandas referentes à população LGBTQIA+ que são perpetuadas pelo preconceito, mas também pela deficiência na construção dos currículos de graduação de saúde nessas singularidades. As próprias diretrizes curriculares nos cursos de Medicina não incorporam a diversidade num contexto plural de sexualidade e gênero na assistência. No intuito de ampliar essas facetas não contempladas, cursos de pós-graduação, eventos acadêmicos, como congressos, seminários, fóruns, capacitações e cursos, acabam sendo o amparo na composição de saberes no que tange à saúde LGBTQIA+ (5).

Nota-se em específico ainda que o curso de graduação de medicina ainda é muito deficitário no ensino e capacitação para abordar as minorias, como é a saúde da população LGBTQIA+, tanto no ciclo clínico quanto nos programas de 
internato e residência, sendo isso corroborado na pesquisa de Negreiros (5), em que diversos médicos entrevistados disseram que, durante toda a sua formação, não tiveram contato com a temática. Dessa forma, temas como gênero e sexualidade são conteúdos muitas vezes apagados da formação ou até mesmo inexistentes, causando um déficit curricular no futuro ao atender esse público e suas necessidades específicas. Assim, abordar os problemas relativos à essa comunidade, seja por meio de eventos, discussões ou reuniões acadêmicas, tem sido uma forma de integração da pauta não heteronormativa nos cursos de saúde, mostrando uma demanda que precisa ser incluída definitivamente nos currículos (6).

Dessa forma, o objetivo do trabalho é relatar a experiência de criação e execução de um evento voltado para a saúde LGBTQIA+, bem como demonstrar a importância e os impactos da discussão dessa pauta na comunidade acadêmica.

\section{RELATO}

O "Proud by Nature - I Fórum de Saúde LGBTQIA+", promovido pela Federação Internacional de Associações de Estudantes de Medicina do Brasil (IFMSA Brazil), comitê da Universidade do Estado do Rio Grande do Norte (UERN), foi um evento realizado totalmente de forma online, sendo uma ação pensada principalmente para profissionais e estudantes das diversas área da saúde a fim de suscitar o debate de uma pauta tão importante e negligenciada que é a saúde dessa comunidade. $O$ evento aconteceu no decorrer do mês de junho de 2020, mês do Orgulho LGBTQIA+ e foi distribuído em quatro encontros. A equipe executora constituiu-se de dois discentes do curso de Medicina que ficaram na coordenação e organização da ação, procurando as principais demandas multidisciplinares na assistência à saúde dessa comunidade. Materiais como a Política Nacional de Saúde Integral LGBT+ do Ministério da Saúde foram consultados e os assuntos escolhidos

Figura 1. Evento

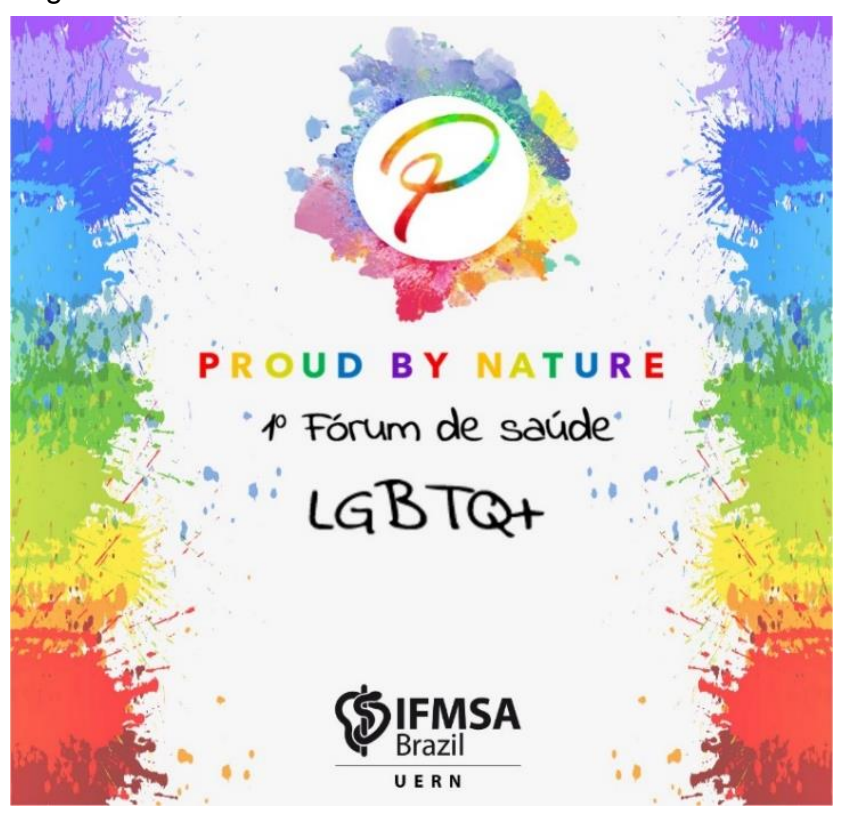

Fonte: autoria própria.
Figura 2. Cronograma do Evento

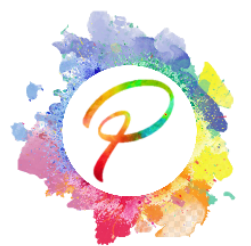

PROUD B Y NATURE

p Fórum de saúde LGBTQ+

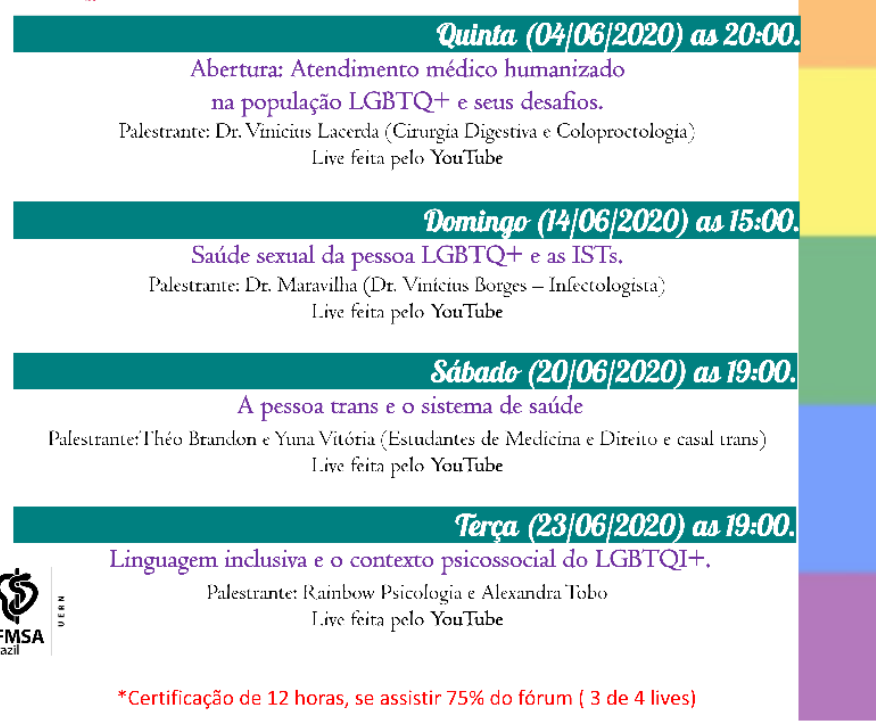

Fonte: Autoria própria.

foram: atendimento humanizado e seus desafios, saúde sexual, saúde da pessoa trans, linguagem inclusiva e saúde psicológica.

Como forma de avaliar o aproveitamento e os benefícios atingidos pelo evento, a coordenação elaborou avaliações de impacto, aplicadas tanto antes do início da programação quanto durante todos os dias de palestras. A aplicação foi por meio de formulário eletrônico, utilizando perguntas de múltipla escolha e em escala Likert. A partir disso, foi possível analisar o aprendizado dos participantes, o grau de satisfação e as principais dificuldades sobre cada tema abordado.

Devido ao contexto da pandemia de SARS-CoV-2 e do isolamento social, a atividade ocorreu de forma online via plataforma de streaming YouTube, no formato de bate-papo entre o palestrante e os coordenadores do evento. Foi possível atingir o maior público possível e criar interação via chat, grupos e redes sociais em geral. O evento não teve nenhuma restrição de acesso ou qualquer tipo de taxa de pagamento, gerando certificado para todos os inscritos que cumpriram $75 \%$ da carga horária assistida. Ainda, contou com mais de 850 inscritos de todo o Brasil interessados em conhecer a realidade e as dificuldades de acesso de diversos grupos não heterocisnormativos, sendo o público principalmente de estudantes dos diversos cursos da área da saúde. Houve intensa divulgação e apoio do evento por instituições e profissionais de saúde do ramo.

O primeiro dia de evento contou com o tema: atendimento médico para a população LGBTQIA+ e seus desafios e foi palestrado pelo médico cirurgião proctologista Vinicius Lacerda. A live abordou, inicialmente, a contextualização da LGBTfobia e a falta de visibilidade e de conhecimento das demandas em saúde dessa comunidade. Em sequência, o 
médico explanou sobre a ausência desse tipo de debate nas universidades, bem como nos espaços de saúde, o que culmina nessa marginalização do conhecimento LGBTQIA+. Ainda, falou-se sobre a importância dos ambulatórios LGBT+ e dos procedimentos transexuais realizados pelo SUS, que são um passo inicial para o avanço. Já o final da live foi voltado para dúvidas sobre sexo anal e seus cuidados básicos, desfazendo muitos mitos e trazendo conhecimentos que muitas vezes não são abordados para profissionais de saúde, possibilitando orientação adequada aos pacientes.

A segunda reunião contou com a presença do infectologista Vinícius Borges, idealizador do canal "Doutor Maravilha", que discutiu com o público sobre a saúde sexual LGBTQIA+ sem tabus. A palestra foi feita com 0 apoio de slides descontraídos que abordaram a empatia com pacientes soropositivos, carga viral indetectável, Profilaxia PréExposição (PrEP) e a Profilaxia Pós-Exposição (PEP), discorrendo sobre os principais mitos ligados ao Vírus da Imunodeficiência Humana (HIV). Ainda, o palestrante falou sobre diagnóstico, prevenção e tratamento das principais Infecções Sexualmente Transmissíveis (ISTs), contextualizando com a vulnerabilidade do público LGBTQIA+ nesse cenário.

Já no terceiro encontro, fez-se a abordagem sobre saúde trans. Os convidados foram um casal transexual, que contaram os desafios enfrentados na busca pela hormonioterapia, o desconhecimento médico sobre as especificidades trans e da transparentalidade, bem como os preconceitos enfrentados no processo de gestação de seu filho biológico, desde o pré-natal a assistência pós-parto. Também se falou da falta do reconhecimento e da representatividade trans dentro dos espaços acadêmicos e aparelhos de saúde, o que acaba por provocar medo nessas pessoas em procurar os serviços.

A última palestra temática discutiu o contexto psicossocial do indivíduo LGBTQIA+ e a linguagem inclusiva. Para tal, os convidados foram a diretora de comunicação e marketing Alessandra Tobo e o psicólogo Hamilton Kida, fundadores de uma rede psicossocial inclusiva que conecta profissionais à comunidade. Dentre os assuntos abordados, discutiu-se a psicologia com consultórios mais acolhedores para este público, as vulnerabilidades psicossociais deste grupo e como a pandemia afetou a saúde mental destes. Ainda, a importância da linguagem neutra, dos pronomes de tratamento adequados à identidade de gênero da pessoa foram colocados como formas de se comunicar com menos preconceito e mais empatia com esse público dentro dos consultórios e da vivência social.

Dentre os pontos positivos, notou-se que o evento pode levar conhecimentos sobre os indivíduos pertencentes à sigla LGBTQIA+ para profissionais e estudantes de saúde e público geral. $\mathrm{E}$ mesmo para indivíduos não heteronormativos, foi uma rica experiência para conhecer sobre todas as letras da sigla. Além disso, a discussão também se mostrou importante por seu caráter informativo: permeou assuntos básicos e complexos relacionados ao sexo saudável entre diversos grupos, de forma didática com auxílio de um coloproctologista que elucidou sobre a forma correta e saudável de fazer a lavagem anal pré-coito (popularmente conhecido como chuca). Através de um profissional infectologista, também foi possível entender melhor sobre as ISTs e as diversas formas de prevenção combinada que são disponibilizadas pelo SUS. Para os estudantes das áreas de saúde, foi imprescindível conhecer as práticas sexuais do grupo e formas de preconceito institucional, tão presentes ainda nos consultórios e clínicas médicas, sendo importante ainda como forma de debate de um assunto nunca em pauta nas aulas dos cursos de medicina no Brasil.

Dentre os pontos negativos, pode-se citar a resistência ainda vigente de muitos discentes e profissionais em se capacitar no atendimento destas populações, o que impacta na adesão de um evento desse eixo. Mas, mesmo com esse entrave, a ação contou com muitos inscritos de todo o Brasil. Quanto à execução do evento em meio a pandemia da COVID-19, destaca-se que 0 formato online requereu novos conhecimentos e habilidades em mídias digitais e plataformas de streaming, exigindo que a coordenação se capacitasse nisso.

\section{DISCUSSÃO}

Inicialmente, é necessária uma análise sobre a grade curricular médica, fundamentada na Diretrizes Curriculares Nacionais (7). Esta, por sua vez, determina o que será ministrado e de que forma. É notório que as diretrizes não estabelecem e não citam, de forma clara, uma base curricular que abarque todas as especificidades envolvidas no atendimento médico diverso e plural de populações não heteronormativas, não fomentando a construção de um pensar médico equânime e humanizado.

Tendo em vista essa invisibilidade institucional, os princípios de cuidado em integralidade, equidade e universalidade do Sistema Único de Saúde (SUS) são prejudicados, pois ao não citar a necessidade do ensino em populações não cisnormativas, faz com que não só o bem-estar físico e mental desse grupo fique prejudicado, mas também estabelece uma relação desigual na estrutura e fluxo de atendimento médico, já que ele se torna excludente (8).

Nesse sentido, fica claro a resistência dos indivíduos LGBTQIA+ ao procurar serviços de saúde, não sendo raros práticas ou comentários preconceituosos, observados em todos os níveis de atendimento ambulatorial, seja ele feito por estudantes ou por profissionais já estabelecidos em suas carreiras. Ao encontrar um serviço, esses indivíduos se deparam com equipes despreparadas e que não fornecem um ambiente respeitoso e acolhedor, carecendo de cuidados enquanto grupo vulnerável e suas necessidades específicas. Assim, a desconfiança e a evasão nos aparelhos de saúde se tornam frequentes (9).

Fazendo um recorte sobre a saúde trans no Brasil, é visível a ausência de travestis e transexuais nos serviços de saúde, principalmente na atenção básica - porta que deveria ser de entrada e permanência desses usuários. Um dos grandes 
motivos é a falta de preparo da equipe multiprofissional em atendê-los, desde o acolhimento até a consulta. Aliado a isso, é preciso assegurar as políticas públicas, que muitas vezes não são seguidas, como exemplo, o uso do nome social que já é um direito no SUS, mas não é colocado em prática (10).

Ainda, por demandarem cuidados específicos, como a hormonioterapia, a população trans têm recorrido aos serviços que foram criados com fins específicos: os ambulatórios LGBTQIA+. No entanto, estes ainda são escassos no Brasil, mostrando a necessidade de ampliar esse atendimento inclusivo (11). Além disso, como explanado durante a terceira palestra do evento sobre saúde trans, a transparentalidade é um desafio na assistência. Em seu espaço de fala, o casal discorreu sobre o preconceito sofrido nos aparelhos de saúde, em fazer seu acompanhamento ginecológico, bem como no pré-natal, onde sofreram com os olhares curiosos de demais pacientes e também de equipes de saúde. Essa assistência carece de capacitação profissional e cria um estigma, que perpetua a transfobia.

Quanto à saúde mental dessa população, há uma vulnerabilidade relativa, que se consolida na psique dessas pessoas devido à exposição a discriminação que sofrem, agressões físicas e verbais, rejeição familiar e social e a necessidade de dissimulação de sua identidade e/ou sexualidade. Todo esse conjunto de violência e sofrimento impactam negativamente na saúde mental LGBTQIA+. Assim, conforme dito na palestra sobre esse tema, é necessário que existam centros psicológicos inclusivos com profissionais aptos à essa demanda.

Ao se referir a sexo seguro e ISTs, destaca-se que, enquanto no mundo os casos de HIV/AIDS sofrem queda nos números nos ultimos anos (12), no Brasil a tendencia é o extremo oposto, sobretudo na população de homens que fazem sexo com homens. Isso se deve principalmente à falta de informação, medo de procurar os serviços de saúde, ser julgado ou sofrer preconceito (13). Apesar de existirem materiais, como o protocolo desenvolvido pelo Departamento Nacional de Vigilância, Prevenção e Controle de Doenças Sexualmente Transmissíveis, HIV/AIDS e Hepatites Virais (DDAHV), falta a divulgação e o engajamento das equipes de saúde em promover essa educação continuada (12). Isso demonstra a baixa adesão às atuais políticas públicas de sexo seguro e prevenção de ISTs no público LGBTQIA+, fatores estes que impactam diretamente no aumento de casos e falta de informação básica, como o acesso à PrEP e a PEP e conhecimentos em prevenção, diagnóstico e tratamento das principais infecções transmitidas por sexo desprotegido.

É evidente o protagonismo da luta LGBTQIA+ na garantia de seus direitos básicos, como a saúde. Os cuidados nesta população estiveram, no Brasil, atrelados quase exclusivamente ao manejo de Infecções Sexualmente Transmissíveis (ISTs), o que acarretou uma discriminação institucional e dos próprios profissionais de saúde. Hoje, entende-se melhor a complexidade dos corpos LGBTQIA+e busca-se melhorar esse atendimento prestado, no entanto, ainda há muito para ser feito neste caminho (3). Desta forma, trazer as demandas desse grupo, tal como foi realizado, é uma forma de quebrar paradigmas e tabus sobre sexualidade na assistência médica.

Assim, encontros como conferências e demais eventos de saúde, são um espaço importante para discussão da pauta LGBTQIA+, apontando a necessidade de incentivar formação e capacitação continuada como alicerce no conhecimento sobre diversidade sexual (2). O incentivo da temática desde a graduação, como é o caso do evento relatado, é de suma importância para provocar o conhecimento e expor o que ainda precisa ser melhorado para um atendimento inclusivo LGBTQIA+ para os profissionais e futuros profissionais de saúde.

A construção do saber médico-científico para a saúde LGBTQIA+ aponta deficiências que vão desde a formação curricular do curso até as especializações e capacitações que deveriam ser ofertadas pelos serviços assistenciais de saúde (5). Notou-se que a maior parte dos participantes do evento era composta de estudantes de medicina, mas contou também com muitos estudantes de outras áreas da saúde. Além disso, muitos nunca tinham entrado em contato com a temática antes do Proud by Nature, o que mostra uma carência de abordagem neste tema. De acordo com a interação do público ouvinte com os palestrantes e os assuntos ministrados, notou-se que houve um grande aprendizado.

Diante da atividade, percebe-se que é fundamental que os cursos e especializações de saúde sejam mobilizados na temática LGBTQIA+. À exemplo, um estudo apresentou uma oficina realizada no internato integrado de Medicina de Família e Comunidade/Saúde Mental na Universidade Federal do Rio de Janeiro (UFRJ). Como resultados da atividade, apontou-se a necessidade de ampliar as discussões em gênero e sexualidade na graduação, residência e como extensões em Medicina, de caráter primordial para garantir os direitos à saúde dessa população, segundo os próprios participantes. Com isso, entende-se a necessidade do conhecimento em saúde LGBTQIA+, a fim de transformar a realidade sociocultural nos ambientes médicos e assistenciais (6).

\section{CONCLUSÃO}

A partir da vivência como coordenação do evento, nota-se quão relevante é tratar da atual negligência à saúde do grupo LGBTQIA+ e realizar programas, eventos e recursos curriculares que tratem cada vez mais do tema. A partir do sucesso de execução do evento, grande adesão do público, interação e impactos positivos de discutir essa temática, os objetivos propostos pelo evento foram cumpridos. Ainda, demonstra-se que as instituições de ensino superior de medicina e suas entidades têm um papel transformador na contribuição não só com a comunidade acadêmica, mas também com o público exterior, espalhando conhecimento, habilidades e competências em conteúdos muitas vezes velados, como é o caso da saúde LGBTQIA+.

Além disso, a possibilidade de o evento ocorrer de forma 
digital ampliou o acesso a palestrantes renomados, público de todos os lugares e tudo isso sem custos. Há perspectivas para que próximas edições do evento aconteçam, com novas óticas. No entanto, embora ações como o Proud by Nature sejam muito válidas para tratar a temática, é importante promover também atividades práticas e interativas para melhor desenvolver os temas, seja em cursos de graduação, instituições de saúde ou residências médicas e multiprofissionais, além de colocar a pauta LGBTQIA+ definitivamente nos seus currículos de saúde, universalizando e afirmando o cuidado assistencial com essa comunidade.

\section{CONFLITO DE INTERESSE}

Os autores declaram que não há potencial conflito de interesse relacionado a esta publicação.

\section{FINANCIAMENTO}

Os autores declaram que não foram utilizados recursos financeiros, patrocínio ou financiamento próprio.

\section{REFERÊNCIAS}

1. Brasil. Constituição (1988). Constituição da República Federativa do Brasil. Brasília, DF: Senado Federal; 1988.

2. da Silva JWSB et al. Políticas públicas de saúde voltadas à população LGBT e à atuação do controle social. Revista de Saúde Pública do Paraná. 2017 Jan; 18(1):140-9.

3. Prado EA, Sousa MF. Políticas públicas e a saúde da população LGBT: uma revisão integrativa. Tempus, actas de saúde colet. 2017 Mar; 11(1): 69-80.

4. Popadiuk GS, Oliveira DC, Signorelli MC. A Política Nacional de Saúde Integral de Lésbicas, Gays, Bissexuais e Transgêneros (LGBT) e o acesso ao Processo Transexualizador no Sistema Único de Saúde (SUS): avanços e desafios. Ciênc. saúde colet. 2017 Maio; 22(5): 1509-19.

5. Negreiros FRN, Ferreira BO, Freitas DN, Pedrosa JIS, Nascimento EF. Saúde de Lésbicas, Gays, Bissexuais, Travestis e Transexuais: da Formação Médica à Atuação Profissional. Rev. bras. educ. med. 2019 JanMar; 43(1):23-31.

6. Loria GB et al. Saúde da população LGBT+ no contexto da atenção primária em saúde: relato de oficina realizada no internato integrado de Medicina de Família e Comunidade/Saúde Mental em uma universidade pública. Rev Bras Med Fam Comunidade. 2019 JanDez;14(41):1807-17.

7. Brasil. Ministério da Educação. Resolução CNE/CES nº 3 , de 20 de Junho de 2014. Diretrizes curriculares nacionais do curso de graduação em medicina. Diário Oficial da União. 23 jun. 2014.
8. Brasil. Lei $n^{\circ} 8.080$, de 19 de setembro de 1990. Dispõe sobre as condições para a promoção, proteção e recuperação da saúde, a organização e o funcionamento dos serviços correspondentes e dá outras providências. Diário Oficial da União. 20 set 1990; Seção 1:018055.

9. Albuquerque MRTC, Botelho NM, Rodrigues CCP. Atenção integral à saúde da população LGBT: Experiência de educação em saúde com agentes comunitários na atenção básica. Rev bras med fam comunidade; 2019 Abril; 14(41):1758-1768.

10. Ferreira BO, Pedrosa JIS, Nascimento EF. Diversidade de gênero e acesso ao sistema único de saúde. Rev Bras Promoç Saúde; 2018 Jan-Mar; 31(1):1-10.

11. Melo DS, Silva BL, Mello R. A sintomatologia depressiva entre lésbicas, gays, bissexuais e transexuais: um olhar para a saúde mental. Rev enferm UERJ; 2019 Out; 27(e41942): p.1-8.

12. Montenegro L, Velasque L, Legrand $S$, Whetten $K$, Mattos R. De, \& Rafael, R. (2019). Public health, HIV care and prevention, human rights and democracy at a crossroad in Brazil. AIDS and Behavior, ANO Mes; 1(4): p-p.

13. Santos ARD, Santos RMM, Souza MLD, Boery RNSDO, Sena ELDS, \& Yarid SD. Implicações bioéticas no atendimento de saúde ao público LGBTT. Revista Bioética (Impressa), 2015 Mai-Ago; 23(2): 400-408. 